\title{
Simulation of photo-excited adenine in water with a hierarchy of equations of motion approach
}

Arend G. Dijkstra, and Valentyn I. Prokhorenko

Citation: The Journal of Chemical Physics 147, 064102 (2017); doi: 10.1063/1.4997433

View online: https://doi.org/10.1063/1.4997433

View Table of Contents: http://aip.scitation.org/toc/jcp/147/6

Published by the American Institute of Physics

\section{Articles you may be interested in}

On the adiabatic representation of Meyer-Miller electronic-nuclear dynamics

The Journal of Chemical Physics 147, 064112 (2017); 10.1063/1.4995301

Coupled electron-nuclear quantum dynamics through and around a conical intersection

The Journal of Chemical Physics 147, 064302 (2017); 10.1063/1.4989780

Tracking an electronic wave packet in the vicinity of a conical intersection

The Journal of Chemical Physics 147, 074101 (2017); 10.1063/1.4989462

Geometric phase effects in excited state dynamics through a conical intersection in large molecules: Ndimensional linear vibronic coupling model study

The Journal of Chemical Physics 147, 064106 (2017); 10.1063/1.4985925

Description of ground and excited electronic states by ensemble density functional method with extended active space

The Journal of Chemical Physics 147, 064104 (2017); 10.1063/1.4996873

Closures of the functional expansion hierarchy in the non-Markovian quantum state diffusion approach

The Journal of Chemical Physics 147, 064113 (2017); 10.1063/1.4986251

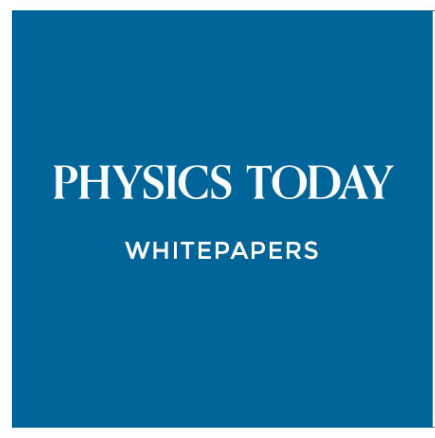

ADVANCED LIGHT CURE ADHESIVES

Take a closer look at what these environmentally friendly adhesive systems can do

\section{READ NOW}

PRESENTED BY

Q.MASTERBOND 


\title{
Simulation of photo-excited adenine in water with a hierarchy of equations of motion approach
}

\author{
Arend G. Dijkstra ${ }^{1, a)}$ and Valentyn I. Prokhorenko ${ }^{2}$ \\ ${ }^{1}$ University of Leeds, School of Chemistry and School of Physics and Astronomy, Leeds LS2 9JT, United Kingdom \\ ${ }^{2}$ Max Planck Institute for the Structure and Dynamics of Matter, Luruper Chaussee 149, \\ 22761 Hamburg, Germany
}

(Received 12 May 2017; accepted 24 July 2017; published online 8 August 2017)

\begin{abstract}
We present a theoretical method to simulate the electronic dynamics and two-dimensional ultraviolet spectra of the nucleobase adenine in water. The method is an extension of the hierarchy of equations of motion approach to treat a model with one or more conical intersections. The application to adenine shows that a two-level model with a direct conical intersection between the optically bright state and the ground state, generating a hot ground state, is not consistent with experimental observations. This supports a three-level model for the decay of electronically excited adenine in water as was previously proposed in the work of V. I. Prokhorenko et al. [J. Phys. Chem. Lett. 7, 4445 (2016)]. Published by AIP Publishing. [http://dx.doi.org/10.1063/1.4997433]
\end{abstract}

\section{INTRODUCTION}

An important goal of spectroscopic and theoretical studies on DNA nucleobases, one of which is adenine, and DNA itself has been the elucidation of the decay mechanism after the absorption of ultraviolet (UV) light. ${ }^{1}$ It has been speculated that DNA is intrinsically photostable due to the presence of ultrafast deactivation channels and that this photostability is important for its biological function as the storage system of the genetic code. ${ }^{2}$

The resolution of the decay mechanism after photoexcitation would be much easier if one could reliably determine the excited state potential energy surfaces. This is in essence a problem of the electronic structure. Several groups have calculated these potential surfaces and their intersections for adenine $;^{3-9}$ however, no consensus has been reached on their exact shape or topology. ${ }^{10}$ Calculations are complex because of the number of electrons that needs to be accounted for, even for a small molecule, the inherent difficulty in reliably computing excited state properties, and the presence of a water solvent in natural systems.

In this situation, it is valuable to derive information from an experiment. Optical measurements directly probe the electronic response of nucleobases after the absorption of light. It turns out that linear UV spectra of these molecules in water are very broad. This can be explained by rapid pure dephasing contributions to the line shape, which basically hides all information about slower relaxation processes. It is then natural to employ nonlinear optical measurements to measure these ultrafast processes. Several groups have been using time-resolved transient absorption (TA) and fluorescence and photoelectron spectroscopies to study the dynamics of excited electrons in nucleobases ${ }^{11-13}$ and larger complexes. ${ }^{14,15}$

a) E-mail: a.g.dijkstra@leeds.ac.uk
Measurements have been interpreted with the help of models that imply the presence of conical intersections between the excited state and the ground state ${ }^{16}$ and of a resultant hot ground state. ${ }^{17}$

Very recently, Prokhorenko et al. ${ }^{18}$ have collected TA and electronic two-dimensional (2D) UV spectra of adenine in water. These measurements offer new information on the electronic excited state dynamics through their extremely high time resolution and through the analysis of the $2 \mathrm{D}$ line shape.

In order to interpret these TA and 2D UV spectra and relate them to the underlying potential energy surfaces, a theoretical method was developed. Here, we will introduce a new technical development in our numerical approach that allows simulations at lower temperatures and compares the calculated spectra for different potential energy surfaces.

The two crucial ingredients of the model are potential energy surfaces that are connected through conical intersections and dissipative dynamics induced by a quantum bath.

Many molecules of interest in spectroscopy exhibit conical intersections. ${ }^{19-26}$ In order to numerically model the electronic dynamics in a system with a conical intersection, one needs to propagate coupled electronic and nuclear degrees of freedom beyond the adiabatic approximation. This approximation clearly breaks down because the energy separation between electronic states becomes arbitrarily small close to the intersection. This makes the calculation of dynamics a difficult problem. Often, the nuclei are treated classically ${ }^{27}$ or the diabatic coupling is treated using perturbation theory. ${ }^{28}$ Stueckelberg oscillations can occur near a conical intersection, ${ }^{29}$ which makes a treatment of the problem even more complicated. Therefore, rich twodimensional spectra are expected for systems with conical intersections. ${ }^{30,31}$

A series of seminal papers on two-dimensional optical spectra of nucleobases was recently presented by Garavelli and 
co-workers. ${ }^{32-35}$ The methodology that was developed by this group combines electronic structure calculations with the sumover-states approach to calculate nonlinear optical signals. In this way, information from quantum chemical computation can be compared directly with the experiment, and predictions on the electronic structure corresponding to a certain nonlinear spectrum can be made.

UV and visible 2D and TA spectroscopic signals were calculated for zero waiting time for several nucleobases, employing complete active space methods to characterize the electronic transitions. ${ }^{32,34}$ Calculations were done in vacuum, and it was shown that the excited state absorption contributions can be used to identify the electronic states in nucleobases. Dimers of stacked nucleobases were also considered. Relaxation mechanisms and their effect on the nonlinear signal were studied as well. ${ }^{33}$ In this study, a QM/MM approach with an explicit solvent was used.

Besides conical intersections, the second crucial ingredient of the model discussed in this work is dissipative dynamics. Numerically exact methods that can be used to calculate nonlinear spectra in this field include the quasiadiabatic path integral (QUAPI) ${ }^{36}$ algorithm and the hierarchy of equations of motion, ${ }^{37}$ an extension of which we will use here. Nonadiabatic coupling between an excited state and the ground state was considered for a one-dimensional model by Tanimura. ${ }^{38}$ In such a one-dimensional model, coupling between diabatic states leads to an avoided crossing of the adiabatic surfaces. To go one step further and properly account for a conical intersection of these surfaces, the standard model needs the introduction of at least two vibrational modes. ${ }^{39}$

In the present work, we aim at interpreting the experimentally measured electronic $2 \mathrm{D}$ spectrum of adenine in water. We use and develop a hierarchy of equations of motion method for potential energy surfaces which cross at conical intersections, which includes effects such as decoherence and dephasing. In addition to the above-mentioned calculations of 2D UV spectra for different potential energy surfaces, we introduce a new procedure to terminate the hierarchy, which improves the numerical efficiency at low temperatures.

\section{MODEL}

Although several excited states play a role in the wavepacket dynamics of photoexcited adenine, ${ }^{40}$ we focus here on a minimal model that corresponds to the kinetic model derived from experimental results. The key aim of our paper is to distinguish between two models- - a two-state model with a direct conical intersection between the optically bright state and the ground state and a three-state model with an intermediate state. We analyse 2D spectra for both models and discuss how nonlinear optical spectroscopy can distinguish between them.

\section{A. Two- and three-state models}

A minimal model for a conical intersection is the linear vibronic coupling model. ${ }^{30}$ In this work, we will compare this model, which has an excited state and a ground state, with a generalization with three potential energy surfaces: the ground state, a bright $\pi \pi^{*}$ state, and a dark state. This model is derived from a kinetic model that can explain the experimental data. $^{18}$

In these models, two types of coordinates play a role: the so-called tuning coordinate, along which the diabatic state energies vary, and the coupling coordinate(s), along which the coupling between diabatic states changes. In the linear vibronic coupling model, the potential energy curves are harmonic along the tuning coordinate, and the dependence of the diabatic coupling on the coupling coordinate is linear.

\section{Two-level model}

The Hamiltonian for the two-level model contains the two electronic states $|g\rangle$ and $|e\rangle$, a tuning mode $Q_{2}$, and a coupling mode $Q_{1}$. It is given by

$$
\begin{aligned}
H_{P}= & \epsilon_{e}|e\rangle\left\langle e\left|+M_{2} \Omega_{2}^{2} Q_{2} d_{e} D_{2}\right| e\right\rangle\langle e| \\
& +M_{1} \Omega_{1}^{2} Q_{1} D_{1}\left(d_{V}|g\rangle\langle e|+\text { h.c. }\right),
\end{aligned}
$$

where $\epsilon_{e}$ is the vertical excitation energy of the excited state and $M_{i}, D_{i}$, and $\Omega_{i}$ are the masses, characteristic length scales, and frequencies of the modes with coordinates $Q_{i}$, and $d_{i}$ are the dimensionless displacements. The potentials for the tuning coordinate $Q_{2}$ and the coupling coordinate $Q_{1}$ are both harmonic.

Damping is included in the model by linear coupling of the system coordinates $Q_{i}$ to a bath of infinitely many harmonic oscillators that are indexed by an index $\alpha$. The interaction of the coupling and tuning modes with the bath is given by the Hamiltonian

$$
\begin{aligned}
H_{S B}= & \sum_{\alpha}\left\{\frac{p_{\alpha}^{2}}{2 m_{\alpha}}+\frac{1}{2} m_{\alpha} \omega_{\alpha}^{2}\left(x_{\alpha}-\frac{g_{1 \alpha}}{m_{\alpha} \omega_{\alpha}^{2}} Q_{1}\right)^{2}\right. \\
& \left.+\frac{1}{2} m_{\alpha} \omega_{\alpha}^{2}\left(x_{\alpha}-\frac{g_{2 \alpha}}{m_{\alpha} \omega_{\alpha}^{2}} Q_{2}\right)^{2}\right\},
\end{aligned}
$$

where $m_{\alpha}, \omega_{\alpha}, x_{\alpha}$, and $p_{\alpha}$ are the masses, frequencies, coordinates, and momenta of the harmonic bath oscillators, and $g_{i \alpha}$ are their coupling strengths to the system.

Because of the assumed linear coupling to a harmonic bath, all necessary information about the bath and the system bath coupling is present in the spectral densities. The spectral densities are defined as

$$
J_{i}(\omega)=\sum_{\alpha} \frac{g_{i \alpha}^{2}}{2 m_{\alpha} \omega_{\alpha}} \delta\left(\omega-\omega_{\alpha}\right) .
$$

In order to be able to treat the coupling and tuning modes as bath modes rather than as system modes and to obtain a practical spectral density for the extended bath, we choose these to be Ohmic,

$$
J_{i}(\omega)=M_{i} \eta_{i} \omega \exp (-\omega / \Lambda),
$$

where we will take the cutoff $\Lambda$ larger than all other frequencies in the system so that it can be set to infinity. The parameters $\eta_{i}$ measure the strength of the damping. The physical picture is that the motion on the harmonic coupling and tuning modes is damped by coupling to the bath of harmonic oscillators, and this damping is Ohmic. The problem is then transformed by including the coupling and tuning modes into the bath. This 
new bath is then affecting the system through a transformed spectral density, which is of the Brownian oscillator type.

Through a canonical transformation, the Hamiltonian is rewritten as ${ }^{41,42}$

$$
\begin{aligned}
H= & \epsilon_{e}|e\rangle\langle e|-\left(d_{e}|e\rangle\langle e|\right) \sum_{\alpha} g_{2 \alpha}^{\prime} x_{\alpha}^{\prime} \\
& -\left(d_{V}|g\rangle\langle e|+\text { h.c. }\right) \sum_{\alpha} g_{1 \alpha}^{\prime} x_{\alpha}^{\prime} .
\end{aligned}
$$

In the following, we will use the notations $H_{S}=\epsilon_{e}|e\rangle\langle e|$, $V_{2}=d_{e}|e\rangle\langle e|$, and $V_{1}=|g\rangle\langle e|+$ h.c. The transformed spectral density, defined for the couplings to the transformed bath $g_{i \alpha}^{\prime}$, is of the Brownian oscillator form.

In the overdamped case, we approximate the spectral density by the Debye form

$$
J_{i \mathrm{OD}}(\omega)=2 \lambda_{i} \omega_{c, i} \frac{\omega}{\omega^{2}+\omega_{c, i}^{2}},
$$

where $\omega_{c, i}$ is the cutoff frequency and $\lambda_{i}$ is the reorganization energy. We note that this approximation is not a formal limit of the Brownian oscillator spectral density, as can be seen from different contributions to the imaginary part of the correlation function (dissipation) as well as to the real part (fluctuations) at low temperatures. The correlation function is connected to the spectral density by the standard formula ${ }^{43}$

$$
C_{i}(t)=\frac{1}{\pi} \int_{0}^{\infty} \mathrm{d} \omega J_{i}(\omega)(\operatorname{coth} \beta \omega / 2 \cos \omega t-i \sin \omega t),
$$

where $\beta=1 / k_{B} T$ is the inverse thermal energy, which is given as the product of the Boltzmann constant $k_{B}$ and the temperature $T$.

\section{Three-level model}

In a similar way, we derive the Hamiltonian for a threelevel model that consists of a ground state, a bright state $|e\rangle$, and a dark state $|d\rangle$. The bright state is connected to the dark state via a conical intersection. Excitations can reach the ground state from the dark state through a second conical intersection. The transformed Hamiltonian is now

$$
\begin{aligned}
H= & \epsilon_{e}|e\rangle\left\langle e\left|+\epsilon_{d}\right| d\right\rangle\langle d|-\left(d_{e}|e\rangle\left\langle e\left|+d_{d}\right| d\right\rangle\langle d|\right) \sum_{\alpha} g_{2 \alpha}^{\prime} x_{\alpha}^{\prime} \\
& -\left(d_{V}|g\rangle\langle d|+\text { h.c. }\right) \sum_{\alpha} g_{1 \alpha^{\prime}}^{\prime} x_{\alpha}^{\prime} \cdot-\left(d_{V^{\prime}}|d\rangle\langle e|+\text { h.c. }\right) \\
& \times \sum_{\alpha} g_{1^{\prime} \alpha^{\prime}}^{\prime} x^{\prime}{ }_{\alpha} .
\end{aligned}
$$

In this model, there are three spectral densities and therefore three correlation functions, one for the tuning mode and one for each of the two coupling modes. The system Hamiltonian is now $H_{S}=\epsilon_{e}|e\rangle\left\langle e\left|+\epsilon_{d}\right| d\right\rangle\langle d|$ and we define $V_{2}=d_{e}|e\rangle\langle e|+$ $d_{d}|d\rangle\left\langle d\left|, V_{1}=d_{V}\right| g\right\rangle\langle d|+$ h.c., and $V_{1^{\prime}}=d_{V^{\prime}}|d\rangle\langle e|+$ h.c.

\section{B. Hierarchy and terminator}

The hierarchy of equations of motion has quickly become the method of choice for simulating non-Markovian quantum dynamics in open quantum systems. Initially proposed by Tanimura and Kubo in $1989,{ }^{37}$ it was developed over the following 20 years. In 2009, the method was applied to photosynthetic light harvesting by Ishizaki and Fleming. ${ }^{44}$ They showed that properly treating the time scale of the environment, which is similar to the typical time scale of the system's dynamics, leads to prolonged quantum coherent oscillations that are missed in simplified treatments of this problem. In recent years, this method has been applied to study various problems such as two-dimensional optical spectroscopy of light harvesting complexes, ${ }^{45}$ DNA, ${ }^{46}$ electron transfer systems, ${ }^{47}$ quantum impurity systems, ${ }^{48}$ and fermionic systems. ${ }^{49}$

The correlation function for the Debye spectral density is of the form

$$
C_{i}(t)=\sum_{k} c_{i k} e^{-\gamma_{i k}|t|} .
$$

For the two-level model, there are two baths, which we labeled $i=1$ and $i=2$, while for the three-level model, there are three baths with $i=1, i=1^{\prime}$ and $i=2$. The sum of exponentials can be obtained by the Matsubara expansion ${ }^{50}$ or by the Padé approximation. ${ }^{51-53}$

The hierarchy of equations of motion is given by ${ }^{54}$

$$
\begin{aligned}
\dot{\rho}^{n}(t)= & -\left(i H_{S}^{\times}+\sum_{i} \sum_{k=0}^{M} n_{i k} \gamma_{i k}\right) \rho^{n}(t) \\
& -\sum_{i}\left(\frac{2 \lambda_{i}}{\beta \omega_{c, i}}-\lambda_{i} \cot \frac{\beta \omega_{c, i}}{2}-\sum_{k=1}^{M} \frac{c_{i k}}{\gamma_{i k}}\right) V_{i}^{\times} V_{i}^{\times} \rho^{n}(t) \\
& -i \sum_{i} \sum_{k=0}^{M} V_{i}^{\times} \rho^{n_{i k}^{+}}(t)-i \sum_{i} \sum_{k=0}^{M} n_{i k}\left(c_{i k} V_{i} \rho^{n_{i k}^{-}}(t)\right. \\
& \left.-c_{i k}^{*} \rho^{n_{i k}^{-}}(t) V_{i}\right) .
\end{aligned}
$$

Here, we use the Matsubara expansion and the notation $\gamma_{i 0}=\omega_{c, i}$. The system Hamiltonian $H_{S}$ and the system-bath coupling operators $V_{i}$ are used as defined above. The notation $V^{\times} A=[V, A]$ denotes the commutator. The index $n$ is a multiindex, which is composed of $2(M+1)$ indices for the two-level model and of $3(M+1)$ indices for the three-level model. All indices are non-negative whole numbers and $M$ is the number of Matsubara frequencies taken into account. The notations $n_{i k}^{+}$ and $n_{i k}^{-}$denote the increase and decrease of the respective index by one. When all the indices are zero, the object $\rho^{n}$ is equal to the physical reduced density matrix. All other matrices $\rho^{n}$, called auxiliary density matrices, are introduced to keep track of the state of the bath and the system-bath correlations. All these matrices are of the same dimension as $H_{S}$.

For deep enough layers of the hierarchy, where $n_{i k} \gamma_{i k}$ is large compared with the typical energy scales in the system Hamiltonian and in the system bath coupling, the Markovian approximation can be made, and the equations of motion can be closed. ${ }^{55,56}$ The terminators are normally applied when $\sum_{i k} n_{i k}>N$ for a chosen $N$. Shi-Yan filtering has been proposed as a way to reduce the size of the hierarchy. ${ }^{57}$ In this paper, we propose and apply a rigorous and efficient termination criterion that uses $\sum_{i k} n_{i k} \gamma_{i k}>\Gamma$, where $\Gamma$ is a convergence parameter that should be chosen to be large compared with the energy scales in the system Hamiltonian and the system bath coupling. Implementation of this criterion results in a much smaller hierarchy than the original criterion. If the number of Matsubara frequencies is included, $M$ is taken large enough 
so that $2(M+1) \pi / \beta>\Gamma$, while if larger Matsubara terms are included in the Markovian approximation, the cutoff energy $\Gamma$ is the only convergence parameter (in addition to the time step). While we use the Matsubara expansion of the correlation function here, this terminator can be applied to other expansions, including the Padé approximation or fitting methods, as well.

2D UV spectra were calculated using the response function formalism, in which the optical pulses are approximated as delta functions in time. Broad band pump-probe spectra were calculated by setting $t_{1}=0$ in the response function.

\section{RESULTS AND DISCUSSION}

\section{A. Linear spectra and the role of low-temperature terms}

At the ground state geometry, where excited states are populated after vertical excitation, the energy gaps between the states are on the order of electron volts. Because it takes some time for the excited state wave packet to reach the conical intersection, the dynamics between states happen on time scales of the order of hundreds of femtoseconds, as is known from the experimental pump probe spectra. Dephasing of the optical coherence, however, happens in tens of femtoseconds, as is clear from the line width of the linear spectrum. Therefore, population relaxation does not play a role in the linear spectrum, which can be understood completely by pure dephasing in a two-level model.

As is shown in Fig. 1, we indeed get a very good fit of the experimental spectrum using a pure dephasing calculation with the Debye spectral density. The temperature was set to $290 \mathrm{~K}\left(\beta=40 \mathrm{eV}^{-1}\right)$. The parameters used in the figure are $\omega_{c, 2}=0.12 \mathrm{eV}$ (fluctuation time scale $34 \mathrm{fs}$ ), $d_{e}$ $=-0.8$, and $\lambda_{2}=0.87 \mathrm{eV}$ (reorganization energy $d_{e}^{2} \lambda_{2}$ $=0.56 \mathrm{eV})$. The reorganization energy that we used here to match the experimental spectrum, of more than half an electron volt, is considerable.

The linear spectrum was calculated directly from the lineshape function, which gives the exact response in the pure dephasing limit. ${ }^{58}$ We found that the inclusion of both

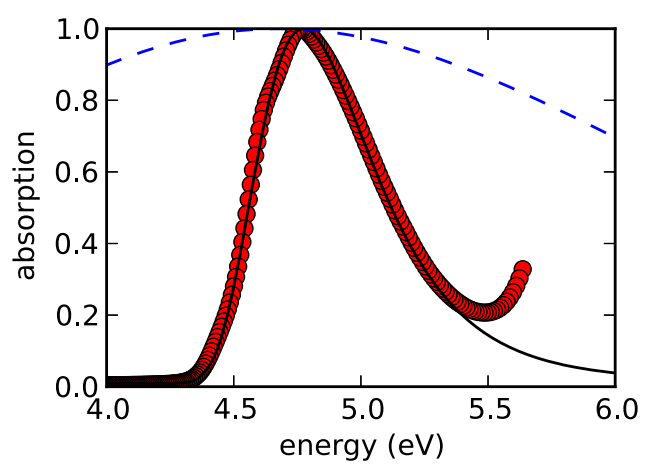

FIG. 1. Calculated and experimental UV absorption spectrum of adenine in water. The red circles are the experimentally observed values, ${ }^{18}$ while the solid line is the simulation. Calculated results used the Drude spectral density with parameters $d_{e}=-0.8, \lambda_{2}=0.87 \mathrm{eV}, \omega_{c, 2}=0.12 \mathrm{eV}$, and a temperature of $290 \mathrm{~K}$. The linear spectrum for the parameters used in Fig. 2 in the pure dephasing limit is shown as a dashed line. dissipation and Matsubara terms is crucial in this parameter regime. The model depends only on the tuning coordinate and does not involve the coupling coordinate(s).

We changed the parameters to understand their effect on the line shape. In brief, larger values of $\omega_{c, 2}$ lead to a more asymmetric line shape. Lowering the temperature also causes this effect, which is in particular visible in the steep rise at the low energy edge of the spectrum. The reorganization energy simply broadens the spectrum. Because the linear spectrum can be fitted with a pure dephasing model and is not sensitive to the population relaxation, it cannot be used to distinguish between the two- and three-level models that give identical linear spectra.

\section{B. Population dynamics and two-dimensional spectra}

The population dynamics of the excited state for the twolevel model is shown in Fig. 4. Calculations were performed at a temperature of $1 / \beta=k_{B} T=0.1 \mathrm{eV}$, and parameters for the tuning mode were obtained by calculating the linear spectrum at this temperature.

In the experimental two-dimensional spectra of nucleobases in water, a strong negative peak is observed, with an intensity that first grows with increasing waiting time and then decreases. ${ }^{18}$ In principle, this observation could be explained by the influence of the hot ground state as was proposed in Ref. 17. The spectra could be fully explained through the twolevel model without any higher lying states that lead to induced absorption. Briefly, in this model, ${ }^{17}$ the initial excitation from the ground state creates a wave packet in the excited state. At a very short waiting time, there are bleaching and stimulated emission contributions from the bright state, leading to a positive peak, but no process that leads to a negative peak. As the waiting time increases, the wave packet relaxes back to the ground state through the conical intersection and forms a hot ground state. The hot ground state is reflected in our model by a time-dependent displacement of the tuning coordinate. Relaxation to the hot ground state followed by absorption to the bright state will lead to a negative peak in the spectrum. This peak is red shifted with respect to the bleaching and stimulated emission because the energy gap between the ground and excited states is smaller when the tuning coordinate is displaced. Clearly, in this model, the negative peak will increase in intensity with waiting time and then decrease and shift to higher energies as the ground state wave packet cools down and the negative peak overlaps with the bleaching and stimulated emission contributions. In this way, this model can qualitatively explain the experimental observations. That this is indeed also the case in our numerical simulations of the calculated 2D UV spectra is shown in Fig. 2. These spectra were calculated using the standard response function approach. ${ }^{58}$ Wide band pump-probe spectra were calculated by setting $t_{1}=0$ when evaluating the response function in the time domain.

However, we observe that the negative peak appears only if the reorganization energy or, equivalently, the displacement of the tuning mode is very large. In this case, the line width of the linear and nonlinear spectra is also too large and incompatible with the experimental data. In fact, the parameters leading 

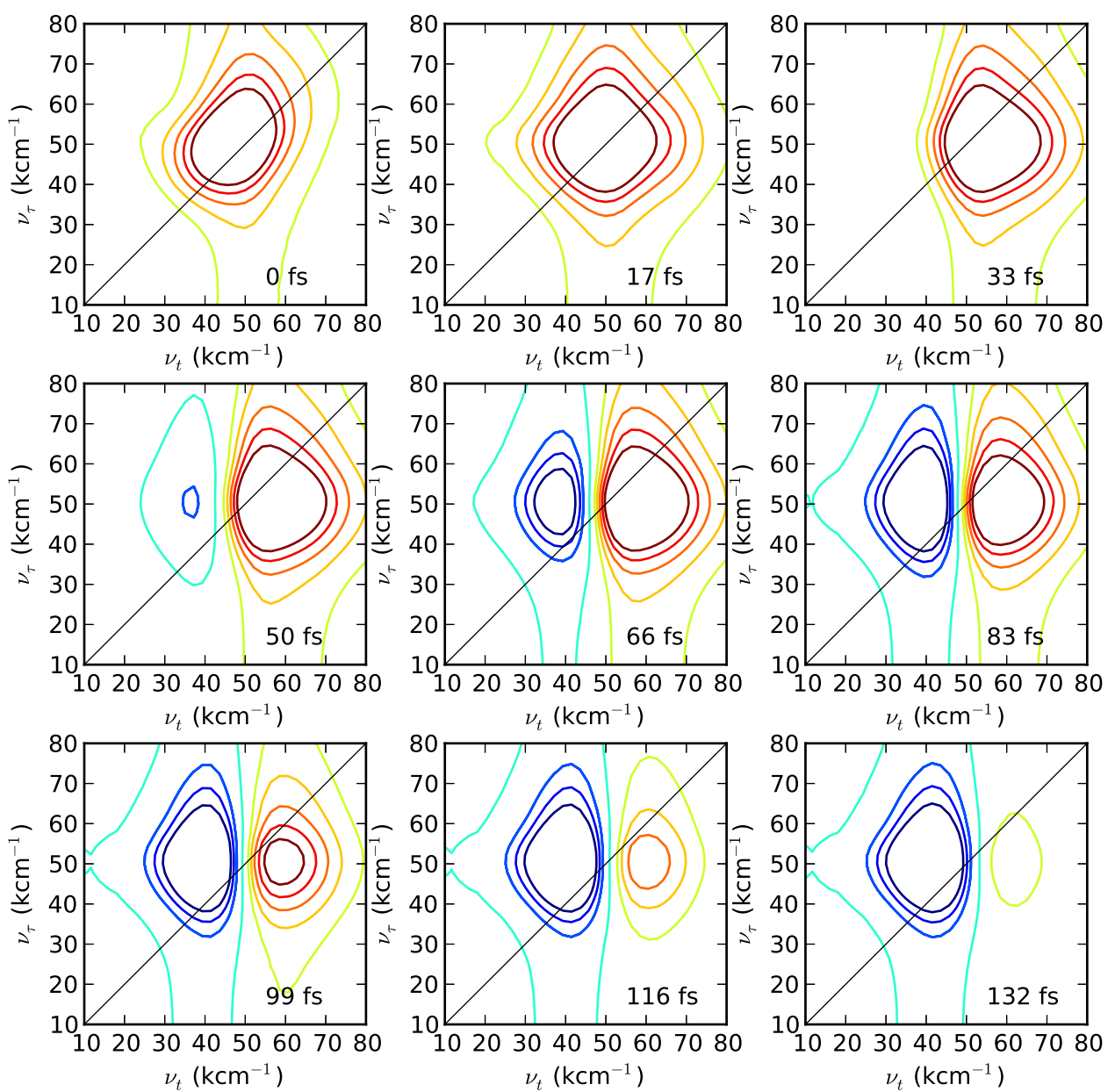

to a good agreement with the experimental spectra do not lead to a negative peak in the two-dimensional spectrum.

This observation is illustrated explicitly in Fig. 3, where we show electronic 2D UV spectra as a function of waiting time with parameters that are in agreement with observed line widths. These parameters also yield population dynamics consistent with the experimentally observed time scales, as is shown in Fig. 4. We see that, apart from small spectral diffusion effects at a short waiting time, the spectral shape is almost independent of the waiting time. This can be explained as follows. If the reorganization energy is small, the ground and excited states are displaced along the tuning coordinate by only a small amount. However, they are offset vertically by the optical excitation energy. The small displacement then leads to a situation where the conical intersection has a larger energy than the vertical excitation energy. Therefore, in these overdamped simulations, the wave packet never reaches the conical intersection and therefore the hot ground state does not form.

From this analysis of the two-level model, we conclude that it cannot explain the experimental observations quantitatively. We considered the possibility that the dissipation of excitation energy strongly increases the local temperature and therefore leads to a value of $\beta$ that increases with time. As long as $\omega_{c}$ and $\lambda$ do not change, this would lead to widening of the pump-probe spectrum with increasing delay time, which is not observed in the experiment.

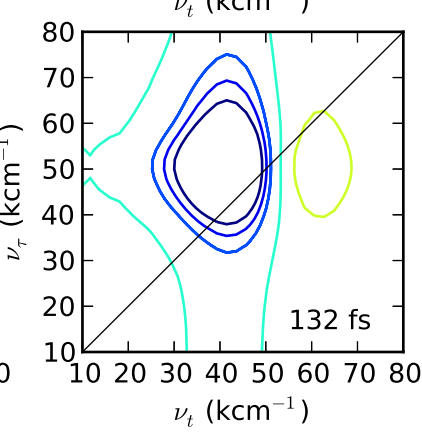

FIG. 2. 2D spectra for the two-level model with parameters $\epsilon_{e}=5.0 \mathrm{eV}$. Coupling mode: $d_{e}=-0.8, \lambda_{2}=2.0 \mathrm{eV}$, $\omega_{c, 2}=0.5 \mathrm{eV}, \beta=1.0 \mathrm{eV}^{-1}$. Tuning mode: $\lambda_{1}=1.0 \mathrm{eV}, d_{V}=0.8, \omega_{c, 1}=0.5$ $\mathrm{eV}, \beta=1.0 \mathrm{eV}^{-1}$. For these parameters, the hot ground state effect leads to a negative peak. However, its line width is much larger than that in the experiment (see Fig. 1), and the time scales of dynamics are much faster.

\section{Three-level model}

By comparing simulated pump probe spectra with the experiment, we find that the bright and dark states must have opposite displacement so that the vertical excitation energy of the dark state is smaller than the vertical excitation energy of the bright state.

We choose the parameters of our model by comparison with the experiment as follows. The product of the reorganization energy for the tuning coordinate and the squared value of the displacement of the bright state is determined by the width of the linear spectrum. The displacement of the dark state is determined from the line widths in the pump probe spectrum. We restrict our parameter set by choosing the damping parameters $\omega_{c, i}$ of all three modes to be equal. The remaining parameters are then the vertical excitation energy of the dark state and the reorganization energy of the coupling modes, which determines the strength of the coupling. We find that these parameters cannot be determined uniquely from the observed spectra but that we can choose them to obtain a reasonable agreement with the experimental data.

As we have shown previously, ${ }^{18}$ simulations with a threelevel model that also includes excited state absorption to higher lying states can well explain the experimental data. In this model, the wave packet relaxes from the bright state to a dark state and then to the ground state. 

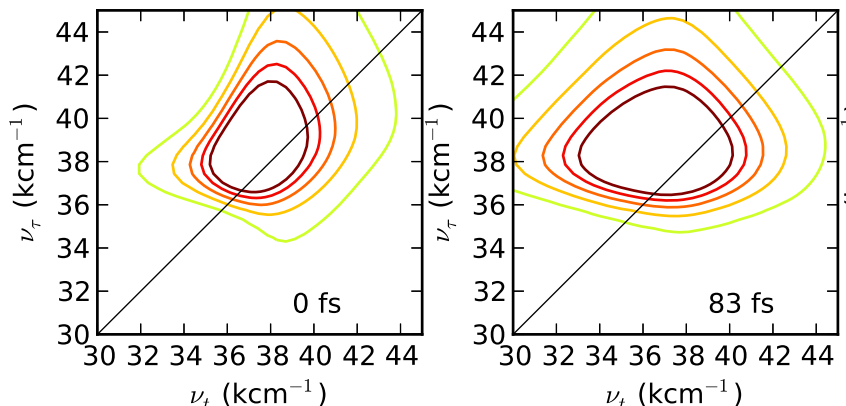

$\nu_{t}\left(\mathrm{kcm}^{-1}\right)$

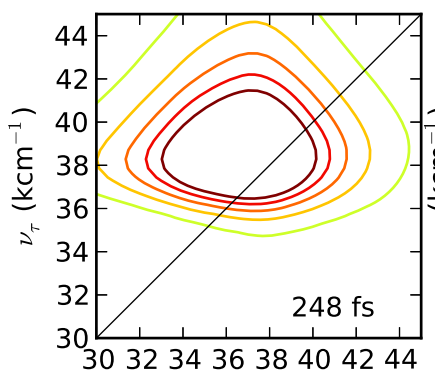

$\nu_{t}\left(\mathrm{kcm}^{-1}\right)$
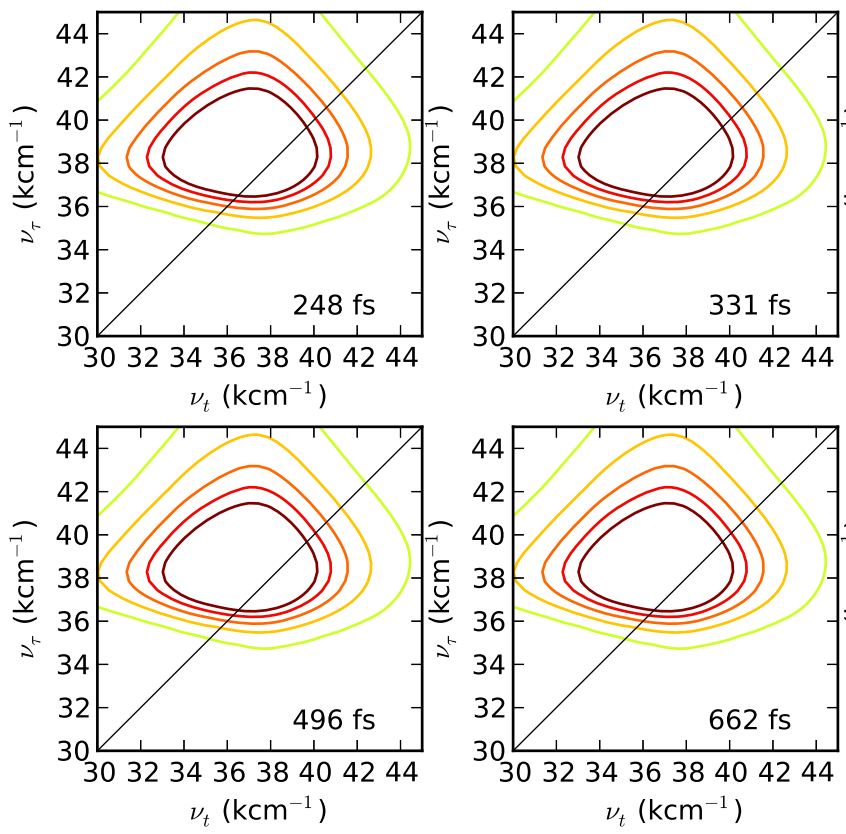

$\nu_{t}\left(\mathrm{kcm}^{-1}\right)$

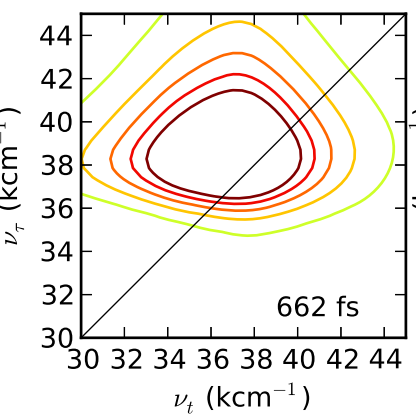

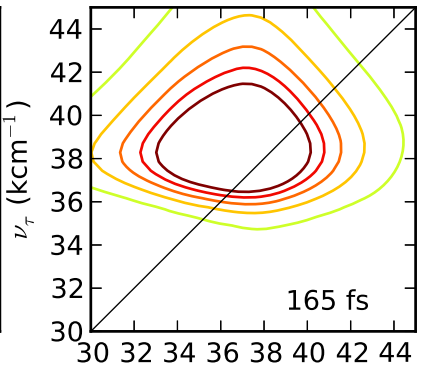

$\nu_{t}\left(\mathrm{kcm}^{-1}\right)$
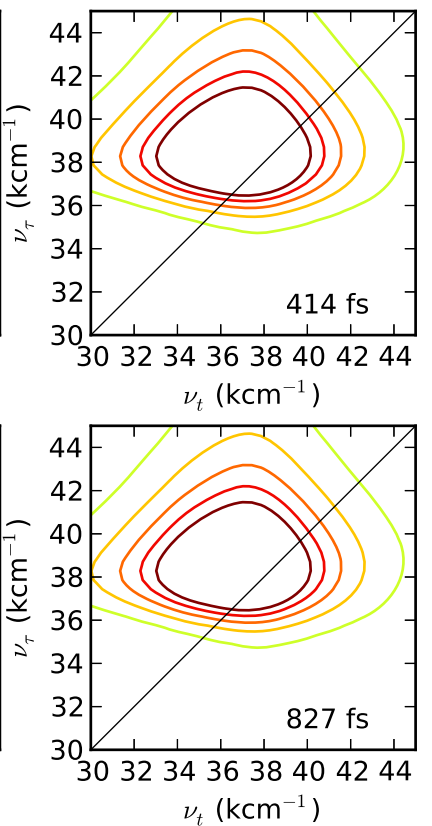

FIG. 3. 2D spectra with the parameters from Fig. 4.
Figure 5 shows the calculated pump-probe and 2D UV spectra for our current three-level model, which was calculated at a much lower (and therefore more realistic) temperature than that in our previous work.

The parameters used in this figure are $\epsilon_{e}=5.0 \mathrm{eV}, \epsilon_{d}$ $=3.5 \mathrm{eV}$, for the tuning mode $d_{e}=-0.8, d_{d}=1.0, \lambda_{2}=0.68 \mathrm{eV}$, and $\omega_{c, 2}=0.3 \mathrm{eV}$. For the coupling mode that connects the bright and dark states, $\lambda_{1^{\prime}} d_{V^{\prime}}^{2}=0.01 \mathrm{eV}$ and $\omega_{c, 1^{\prime}}=0.3 \mathrm{eV}$; for

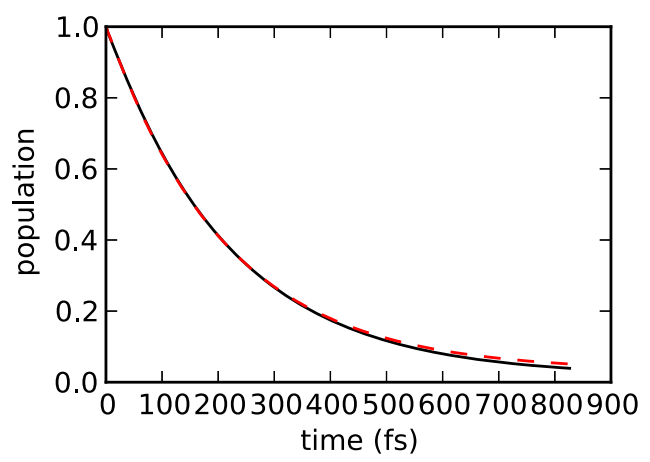

FIG. 4. Dynamics for the two-level system with the parameters: coupling mode $\lambda_{1}=0.1 \mathrm{eV}, \omega_{c, 1}=0.3 \mathrm{eV}, \beta=10.0 \mathrm{eV}^{-1}, d_{V}=0.8$; tuning mode: $\lambda_{2}=0.68 \mathrm{eV}, \omega_{c, 2}=0.3 \mathrm{eV}, \beta=10.0 \mathrm{eV}^{-1}, d_{e}=-0.8$. (Solid line) $\Gamma=6 \mathrm{eV}, 10$ Matsubara frequencies are included, and (dashed line) $\Gamma=5 \mathrm{eV}$, 8 Matsubara frequencies. Plotted is the excited state population. the coupling mode that connects the dark state to the ground state, $\lambda_{1} d_{V}^{2}=0.005 \mathrm{eV}$ and again $\omega_{c, 1}=0.3 \mathrm{eV}$. The transition dipole moment from the dark state to doubly excited states was set to 1.4 , while induced absorption from the bright state was not taken into account. The potential energy surfaces for the ground, bright, and dark states are shown in Fig. 6. Note

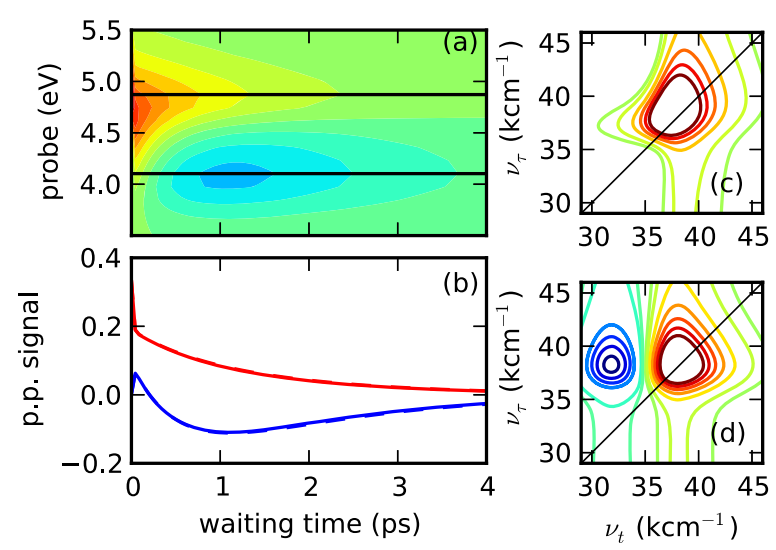

FIG. 5. Calculated pump-probe and 2D UV spectra for the three-level model. (a) Broad band pump probe spectrum. (b) Cuts through the pump-probe spectrum at the energies indicated with black lines in panel (a). Solid and dashed curves show that convergence was reached with respect to $\Gamma$. (c) $2 \mathrm{D}$ UV spectrum at zero waiting time. (d) $2 \mathrm{D}$ UV spectrum at a waiting time of $517 \mathrm{fs}$. 


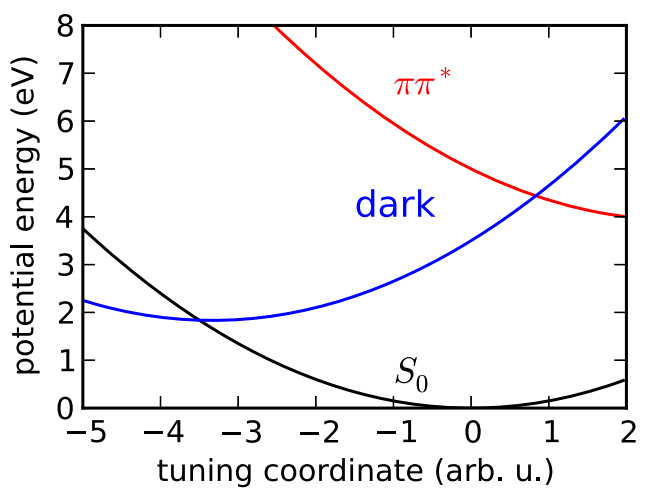

FIG. 6. Model potential energy surfaces for the three-level model used in the present calculation.

that the frequency of the harmonic potentials does not appear explicitly in the theoretical model in the overdamped limit that we employ here.

The solid and dashed lines in Fig. 5 show the results for $\Gamma=5.0 \mathrm{eV}$ and $\Gamma=4.0 \mathrm{eV}$. They almost completely overlap showing that convergence was reached.

The inverse temperature in these calculations was set to $\beta=10 \mathrm{eV}^{-1}\left(k_{B} T=0.1 \mathrm{eV}\right)$. This is still much higher than room temperature but far in the low temperature regime compared with the vertical excitation energy of the bright state, as can be seen by evaluating $\beta \epsilon_{e}=50$, which is much larger than 1 , and also in the low temperature regime compared with the energy difference between the bright state and the dark state, $\beta\left(\epsilon_{e}-\epsilon_{d}\right)=15$.

From these simulations on the three-level system, we conclude that it can fully explain the experimental data.

\section{Excited state coherence in a dimer}

In this section, we briefly present results that go beyond the single nucleobase studied so far and observe a dimer, which is a model of two stacked adenine bases. In order to study the effect of pure dephasing on the decay of the coherence between the bases, we consider coupled two-level systems. We set $\omega_{c}=0.12 \mathrm{eV}, \lambda d_{e}^{2}=0.56 \mathrm{eV}$, and a resonant transfer interaction between the two monomers of $0.1 \mathrm{eV}$. We then calculate the resulting time dependence of the inter-base coherence for various temperatures. The coherence is defined as the magnitude of the off-diagonal element of the density matrix for the singly excited state. A coherent superposition of two excited monomers, as is prepared by optical excitation, initially loses coherence very quickly, on a time scale around $10 \mathrm{fs}$. However, significant coherence remains at much longer times, which is stable against pure dephasing at low temperatures. Dephasing due to population relaxation will eventually destroy this coherence, but this happens on the times scale of 100s of femtoseconds. These findings are illustrated in a numerical calculation in Fig. 7. The relevance of these time scales can be seen by comparing them with the typical time scale for the energy transport between two monomers. Coherent energy transfer occurs on a time scale dictated by $1 / J$, where $J$ is the resonant transfer coupling. For nucleobases, $J$ is a few hundred wavenumbers. ${ }^{59}$ This corresponds to time scales of around $100 \mathrm{fs}$. Therefore,

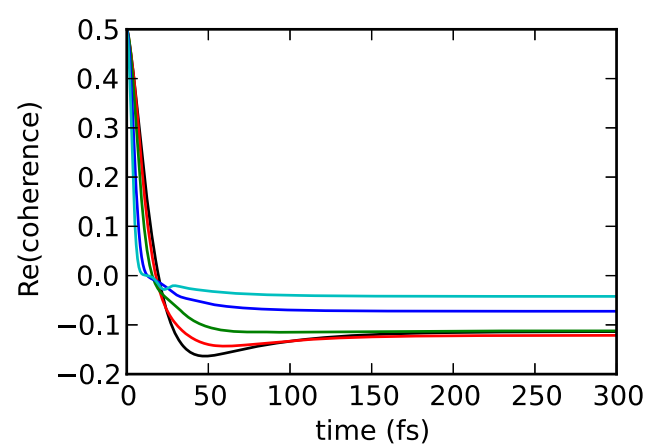

FIG. 7. Coherence (see the main text) between two molecules in a dimer for different temperatures. (cyan) $\beta=1$, (blue) 2, (green) 5, (red) 10 , (black) $20 \mathrm{eV}^{-1}$. Lower temperature leads to a larger value of the steady state coherence.

we expect some degree of exciton effects in stacked adenine bases.

\section{CONCLUSION}

In this paper, we have compared two- and three-level models for the photophysics of adenine in water. Based on calculated two-dimensional UV spectra, we have shown that harmonic two-level models cannot explain the experimental spectra.

While a three-level model with a dark state fully explains the experimentally observed negative peak in the $2 \mathrm{D} \mathrm{UV}$ spectra and the time dependence of the TA spectrum, we cannot rule out that the dark state is in fact the same state as the bright state. In this case, the transition dipole to the ground state must decrease strongly along the tuning coordinate (non-Condon behaviour), while a strong transition to high-lying excited states is possible. This point requires further investigation.

Our work can be extended in several ways. Since the reorganization energy of the coupling modes is small, one may consider treating them perturbatively while including the tuning mode through a polaron transformation. ${ }^{28}$ The method that we use is not limited to overdamped dynamics on the potential energy curves but can also be used with underdamped vibrations. ${ }^{60}$ Finally, based on our finding that some coherent energy transport is possible, it will be interesting to investigate nucleobase oligomers. In such simulations, one should also consider possible intramolecular charge transfer states.

We expect that our work will generate new interest in the connection between $a b$ initio calculated potential energy surfaces, fluctuations, and nonlinear optical spectra.

\section{ACKNOWLEDGMENTS}

A.G.D. thanks Jason Biggs and Tatsushi Ikeda for helpful discussions. During the initial stages of this work, A.G.D. was supported by a Marie Curie International Incoming Fellowship within the 7th European Community Framework Programme (Grant No. 627864). Some of this work was undertaken on ARC2, part of the High Performance Computing facilities at the University of Leeds, UK. Simulation data are available at https://doi.org/10.5518/191. 
${ }^{1}$ C. E. Crespo-Hernández, B. Cohen, P. M. Hare, and B. Kohler, Chem. Rev. 104, 1977 (2004).

${ }^{2}$ T. Fiebig, J. Phys. Chem. B 113, 9348 (2009).

${ }^{3}$ A. L. Sobolewski and W. Domcke, Eur. Phys. J. D 20, 369 (2002).

${ }^{4}$ L. Blancafort, J. Am. Chem. Soc. 128, 210 (2006).

${ }^{5}$ E. Fabiano and W. Thiel, J. Phys. Chem. A 112, 6859 (2008).

${ }^{6} \mathrm{M}$. Barbatti and H. Lischka, J. Am. Chem. Soc. 130, 6831 (2008).

${ }^{7}$ M. Barbatti, A. J. Aquino, J. J. Szymczak, D. Nachtigallová, P. Hobza, and H. Lischka, Proc. Natl. Acad. Sci. U. S. A. 107, 21453 (2010).

${ }^{8}$ M. Barbatti, Z. Lan, R. Crespo-Otero, J. J. Szymczak, H. Lischka, and W. Thiel, J. Chem. Phys. 137, 22A503 (2012).

${ }^{9}$ Y. Lei, S. Yuan, Y. Dou, Y. Wang, and Z. Wen, J. Phys. Chem. A 112, 8497 (2008).

${ }^{10}$ S. Mai, M. Richter, P. Marquetand, and L. González, Top. Curr. Chem. 355, 99 (2015).

${ }^{11}$ T. Gustavsson, A. Sharonov, D. Onidas, and D. Markovitsi, Chem. Phys. Lett. 356, 49 (2002).

${ }^{12}$ W.-M. Kwok, C. Ma, and D. L. Phillips, J. Am. Chem. Soc. 128, 11894 (2006).

${ }^{13}$ C. Z. Bisgaard, H. Satzger, S. Ullrich, and A. Stolow, ChemPhysChem 10, 101 (2009).

${ }^{14}$ C. E. Crespo-Hernández, B. Cohen, and B. Kohler, Nature 436, 1141 (2005).

${ }^{15}$ I. Buchvarov, Q. Wang, M. Raytchev, A. Trifonov, and T. Fiebig, Proc. Natl. Acad. Sci. U. S. A. 104, 4794 (2007).

${ }^{16} \mathrm{~S}$. Brøndsted Nielsen and T. I. Sølling, ChemPhysChem 6, 1276 (2005)

${ }^{17}$ P. M. Hare, C. E. Crespo-Hernández, and B. Kohler, Proc. Natl. Acad. Sci. U. S. A. 104, 435 (2006).

${ }^{18}$ V. I. Prokhorenko, A. Picchiotti, M. Pola, A. G. Dijkstra, and R. J. D. Miller, J. Phys. Chem. Lett. 7, 4445 (2016).

${ }^{19}$ D. Polli, P. Altoè, O. Weingart, K. M. Spilane, C. Manzoni, D. Brida, G. Tomasello, G. Orlandi, P. Kukura, R. A. Mathies et al., Nature 467, 440 (2010)

${ }^{20}$ G. A. Worth and L. S. Cederbaum, Annu. Rev. Phys. Chem. 55, 127 (2004).

${ }^{21}$ M. J. Bearpark, F. Bernardi, S. Clifford, M. Olivucci, M. A. Robb, B. R. Smith, and T. Vreven, J. Am. Chem. Soc. 118, 169 (1996).

${ }^{22}$ L. Joubert-Doriol, I. G. Ryabinkin, and A. F. Izmaylov, J. Chem. Phys. 139, 234103 (2013).

${ }^{23}$ M. Kowalewski, K. Bennett, K. E. Dorfman, and S. Mukamel, Phys. Rev. Lett. 115, 193003 (2015).

${ }^{24}$ M. Kowalewski and S. Mukamel, J. Chem. Phys. 143, 044117 (2015).

${ }^{25}$ R. Szabla, D. Tuna, R. W. Góra, J. Šponer, A. L. Sobolewski, and W. Domcke, J. Phys. Chem. Lett. 4, 2785 (2013).

${ }^{26}$ K. Saita, M. G. D. Nix, and D. V. Shalashilin, Phys. Chem. Chem. Phys. 15, 16227 (2013).

${ }^{27}$ J. P. Malhado, M. J. Bearpart, and J. T. Hynes, Front. Chem. 2, 97 (2014).

${ }^{28}$ A. F. Izmaylov, D. Mendive-Tapia, M. J. Bearpark, M. A. Robb, J. C. Tully, and M. J. Frisch, J. Chem. Phys. 135, 234106 (2011).
${ }^{29}$ P. Nalbach, S. Javanbakht, C. Stahl, and M. Thorwart, "Stueckelberg oscillations in a two-state two-path model of a conical intersection," Ann. Phys. (published online).

${ }^{30}$ J. Krčmáŕ, M. F. Gelin, D. Egorova, and W. Domcke, J. Phys. B: At., Mol. Opt. Phys. 47, 124019 (2014).

${ }^{31}$ M. Sala and D. Egorova, Chem. Phys. 481, 206 (2016).

${ }^{32}$ A. Nenov, A. Giussani, J. Segarra-Martí, V. K. Jaiswal, I. Rivalta, G. Cerullo, S. Mukamel, and M. Garavelli, J. Chem. Phys. 142, 212443 (2015).

${ }^{33}$ A. Nenov, J. Segarra-Marti, A. Giussani, I. Conti, I. Rivalta, E. Dumont, V. K. Jaiswal, S. Altavilla, and S. G. M. Mukamel, Faraday Discuss. 177, 345 (2015).

${ }^{34}$ A. Giussani, J. Segarra-Martí, A. Nenov, I. Rivalta, A. Tolomelli, S. Mukamel, and M. Garavelli, Theor. Chem. Acc. 135, 121 (2016).

${ }^{35}$ I. Conti, A. Nenov, S. Hoefinger, S. F. Altavilla, I. Rivalta, E. Dumont, G. Orlandi, and M. Garavelli, Phys. Chem. Chem. Phys. 17, 7291 (2015).

${ }^{36}$ N. Makri, J. Phys. Chem. A 102, 4414 (1998).

${ }^{37}$ Y. Tanimura and R. Kubo, J. Phys. Soc. Jpn. 58, 101 (1989).

${ }^{38}$ Y. Tanimura, J. Chem. Phys. 137, 22A550 (2012).

${ }^{39}$ W. Domcke, D. R. Yarkony, and H. Köppel, Conical Intersections: Theory, Computation and Experiment (World Scientific, 2011).

${ }^{40}$ W. M. I. Hassan, W. C. Chung, N. Shimakura, S. Koseki, H. Kono, and Y. Fujimura, Phys. Chem. Chem. Phys. 12, 5317 (2010).

${ }^{41}$ A. Garg, J. N. Onuchic, and V. Ambegaokar, J. Chem. Phys. 83, 4491 (1985).

${ }^{42}$ M. Tanaka and Y. Tanimura, J. Chem. Phys. 132, 214502 (2010).

${ }^{43}$ U. Weiss, Quantum Dissipative Systems (World Scientific, 2008).

${ }^{44}$ A. Ishizaki and G. R. Fleming, Proc. Natl. Acad. Sci. U. S. A. 106, 17255 (2009).

${ }^{45}$ C. Kreisbeck and T. Kramer, J. Phys. Chem. Lett. 3, 2828 (2012).

${ }^{46}$ A. G. Dijkstra and Y. Tanimura, New J. Phys. 12, 055005 (2010).

${ }^{47}$ M. Tanaka and Y. Tanimura, J. Phys. Soc. Jpn. 78, 073802 (2009).

${ }^{48}$ Y. Cheng, W. Hou, Y. Wang, Z. Li, J. Wei, and Y. Yan, New J. Phys. 17, 033009 (2015).

${ }^{49}$ J. S. Jin, X. Zheng, and Y. J. Yan, J. Chem. Phys. 128, 234703 (2008).

${ }^{50}$ Y. Tanimura, J. Phys. Soc. Jpn. 75, 082001 (2006).

${ }^{51}$ J. Hu, R.-X. Xu, and Y. Yan, J. Chem. Phys. 133, 101106 (2010).

${ }^{52}$ B.-L. Tian, J.-J. Ding, R.-X. Xu, and Y. Yan, J. Chem. Phys. 133, 114112 (2010).

${ }^{53}$ J. Hu, M. Luo, F. Jiang, R.-X. Xu, and Y. Yan, J. Chem. Phys. 134, 244106 (2011).

${ }^{54}$ A. G. Dijkstra and Y. Tanimura, Phys. Rev. Lett. 104, 250401 (2010).

${ }^{55}$ A. Ishizaki and Y. Tanimura, J. Phys. Soc. Jpn. 74, 3131 (2005).

${ }^{56}$ A. Ishizaki and G. R. Fleming, J. Chem. Phys. 130, 234111 (2009).

${ }^{57}$ Q. Shi, L. Chen, G. Nan, R.-X. Xu, and Y. Yan, J. Chem. Phys. 130, 084105 (2009).

${ }^{58}$ S. Mukamel, Principles of Nonlinear Optical Spectroscopy (Oxford University Press, 1995).

${ }^{59}$ A. Czader and E. R. Bittner, J. Chem. Phys. 128, 035101 (2008).

${ }^{60}$ H.-G. Duan, R. J. D. Miller, and M. Thorwart, J. Phys. Chem. Lett. 7, 3491 (2016). 